\title{
ENFERMEDADES GENÉTICAS: XERODERMA PIGMENTOSO. AMOR A MEDIA NOCHE (2017)
}

Genetic Diseases: Xeroderma pigmentosum. Midnight Sun (2017)

Juan Carlos ROMERO-BENAVIDES ${ }^{1}$; Natalia BAILON-MOSCOSO²

${ }^{1}$ Departamento de Química y Ciencias Exactas. Universidad Tecnica Particular de Loja (Ecuador). ${ }^{2}$ Departamento de Ciencias de la Salud. Universidad Tecnica Particular de Loja (Ecuador).

Correo electrónico: ncbailon@utpl.edu.ec

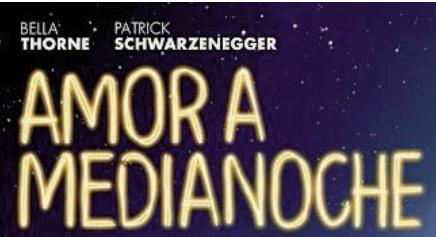

LOS SUEÑOS SE HACEN REALIDAD DE NOCHE

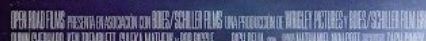

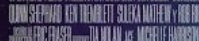

gar

PRÓXIMAMENTE a

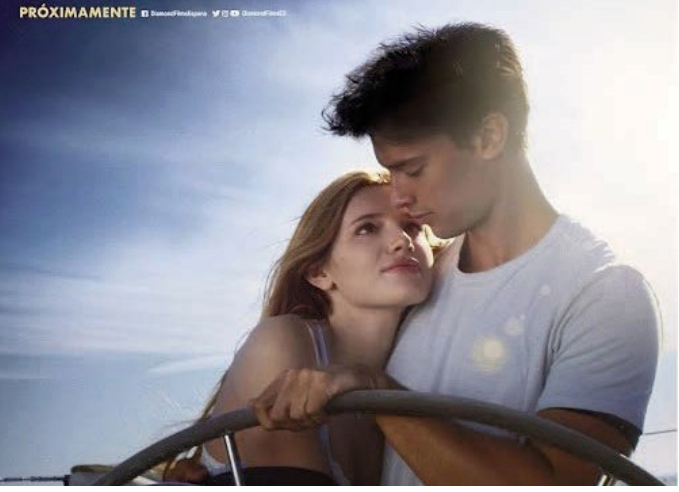

Acción: EE. UU., época actual. Cartel español.
Título original: Midnight Sun.

País: EE. UU.

Año: 2017.

Director: Scott Speer.

Música: Nate Walcott.

Fotografía: Ruben Impens.

Montaje: Alain Dessauvage.

Guion: Eric Kirsten, basada en la película Taiyou no Uta de Kenji Bandō.

Intérpretes: Bella Thorne, Patrick Schwarzenegger, Quinn Shephard, Tiera Skovbye, Jaeda Lily Miller, Kiefer O’Reilly, Allyson Grant, Dean Petriw, Ava Dewhurst, Rob Riggle, Ken Tremblett, Jenn Griffin, Paul McGillion, ...

Color: color.

Duración: 91 minutos.

Género: drama romántico.

Idioma original: inglés.

Productoras: Wrigley Pictures, Boies, Schiller Film Group.

Sinopsis: «Basada en la película japonesa del 2006 del mismo nombre se centra en Katie, una joven de 17 años de edad, encerrada desde la infancia en su casa durante el día por una rara enfermedad que hace que incluso la mínima cantidad

Rev. Med. Cine. 2020; 16(3), 235-238 Ediciones Universidad de Salamanca / @@@ J. Med. Mov., 2020; 16 (3), $235-238$ 
de luz solar sea mortal. El destino interviene cuando conoce a Charlie y se embarcan en un romance de verano (FILMAFFINITY)».

Disponibilidad: Amor a medianoche (DVD). Diamond Films España; 2018.

\section{Enlaces:}

https://www.filmaffinity.com/es/film929042. html

ht tps://www.imdb.com/title/ tt4799066/?ref_=nv_sr_srsg_0

\section{Tráiler en español}

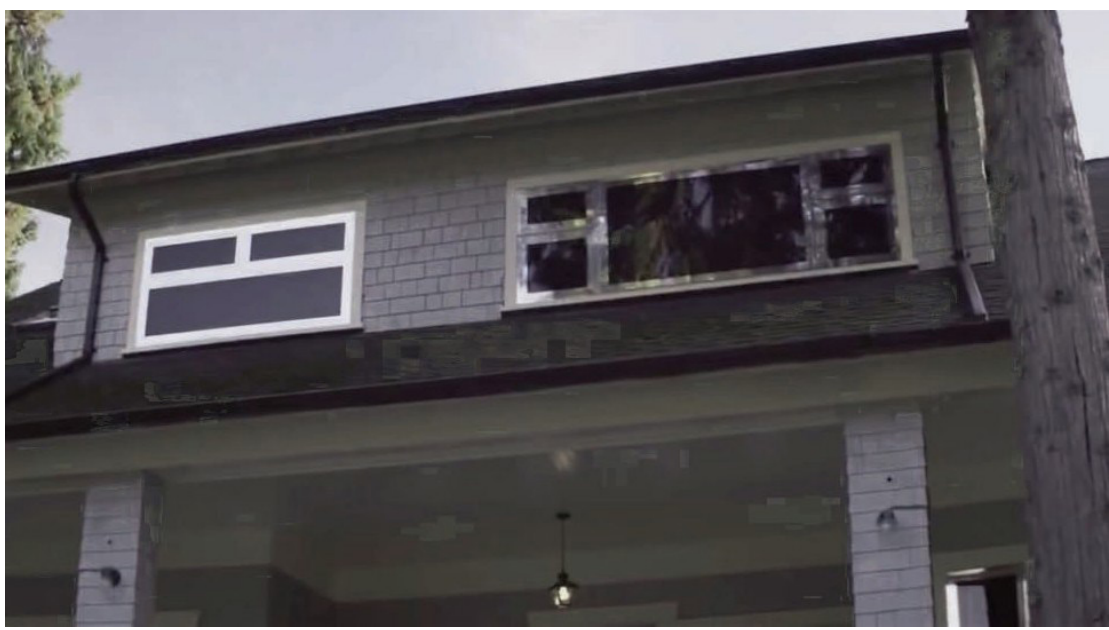

Protecciones especiales en la casa para evitar el ingreso de los rayos ultravioletas.

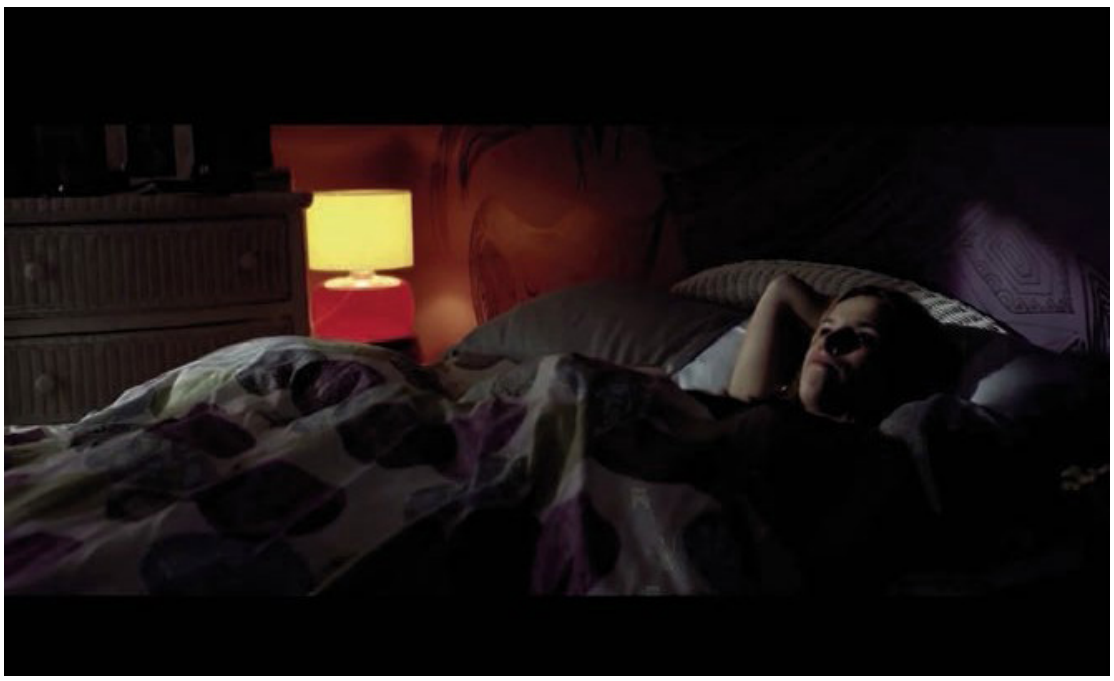

Cambios de hábitos de vida, duermen durante el día y generan actividades por la noche. 


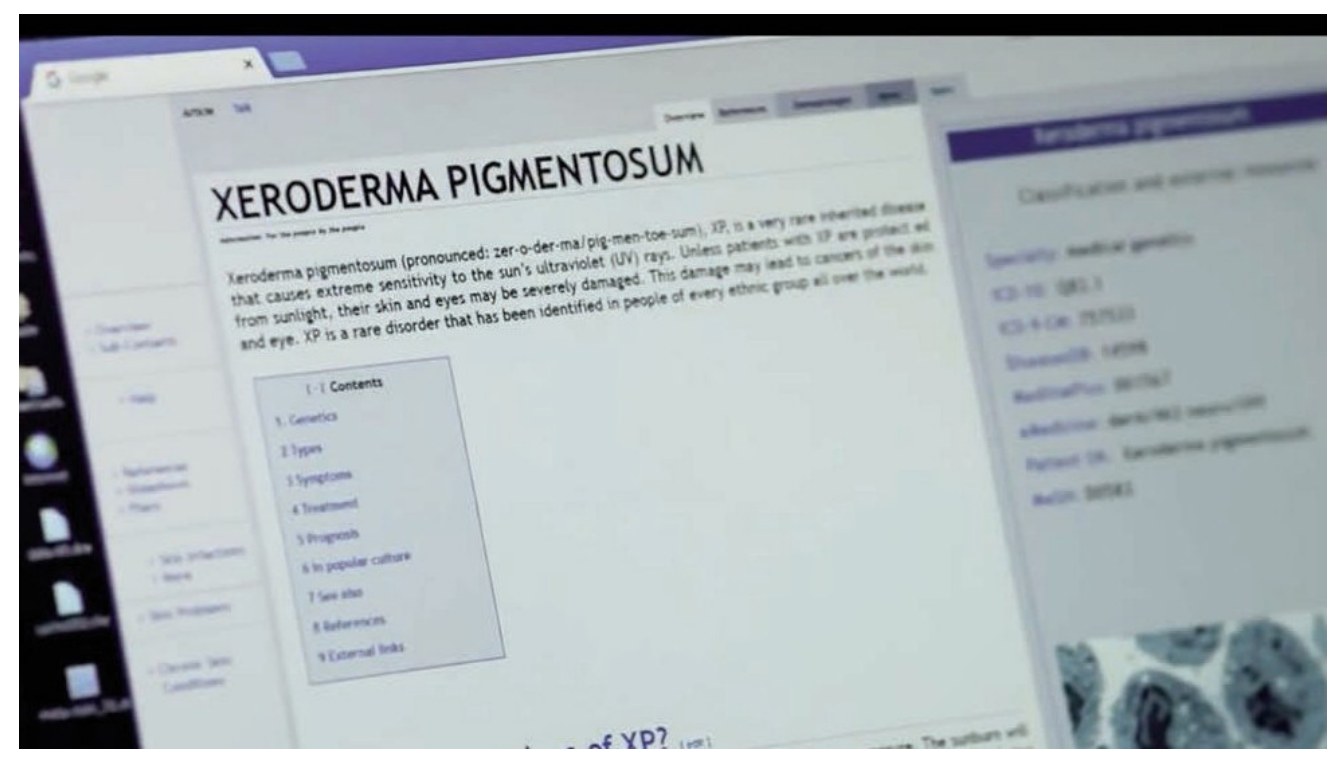

Descripciones acerca de la enfermedad.

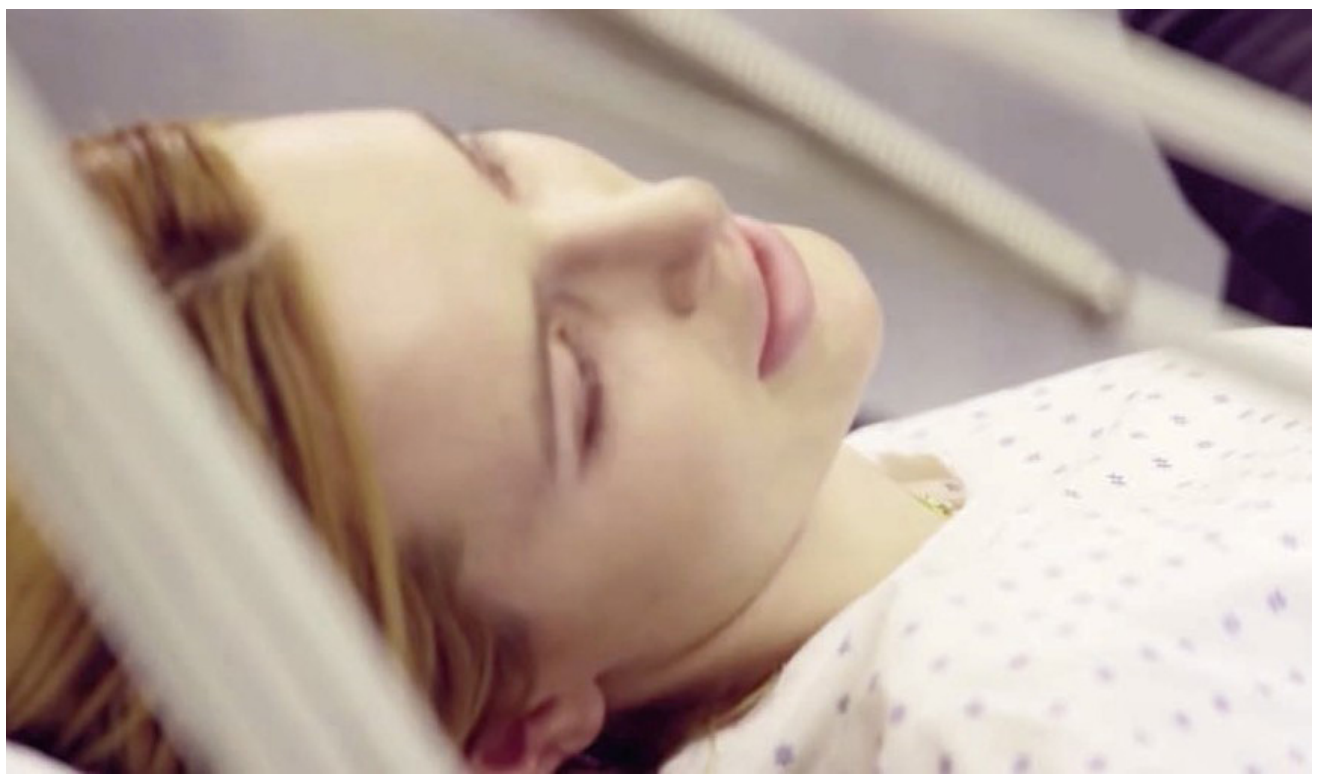

Controles periódicos con el fin de detectar cáncer de piel.

Rev. Med. Cine. 2020; 16(3), 235-238 Ediciones Universidad de Salamanca / @®@ J. Med. Mov., 2020; 16 (3), $235-238$ 
ENFERMEDADES GENÉTICAS: XERODERMA PIGMENTOSO. AMOR A MEDIA NOCHE (2017) JUAN CARLOS ROMERO-BENAVIDES; NATALIA BAILON-MOSCOSO

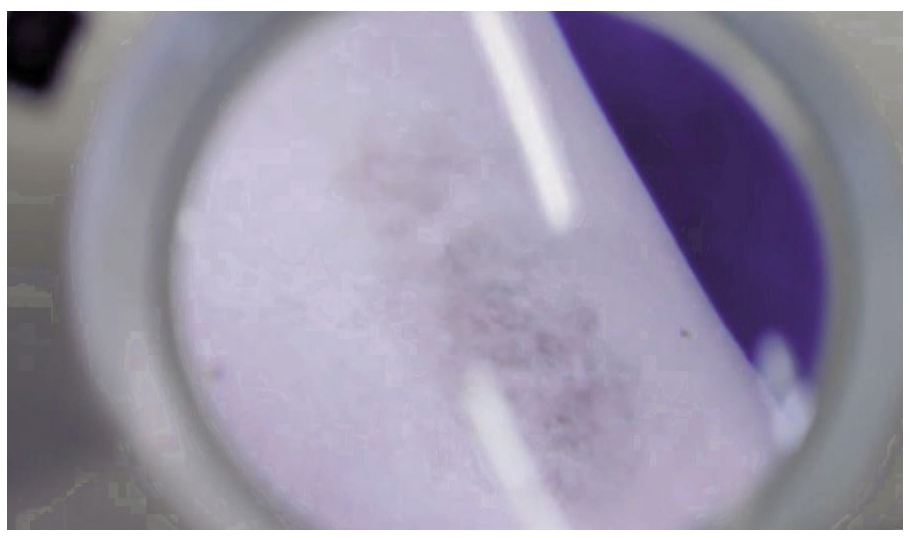

Daños en la piel.

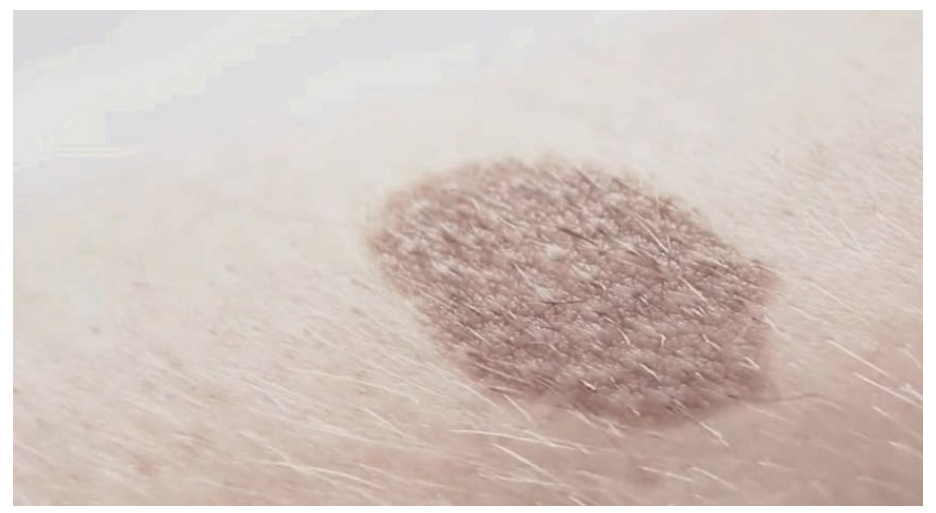

Desarrollo de cáncer de piel.

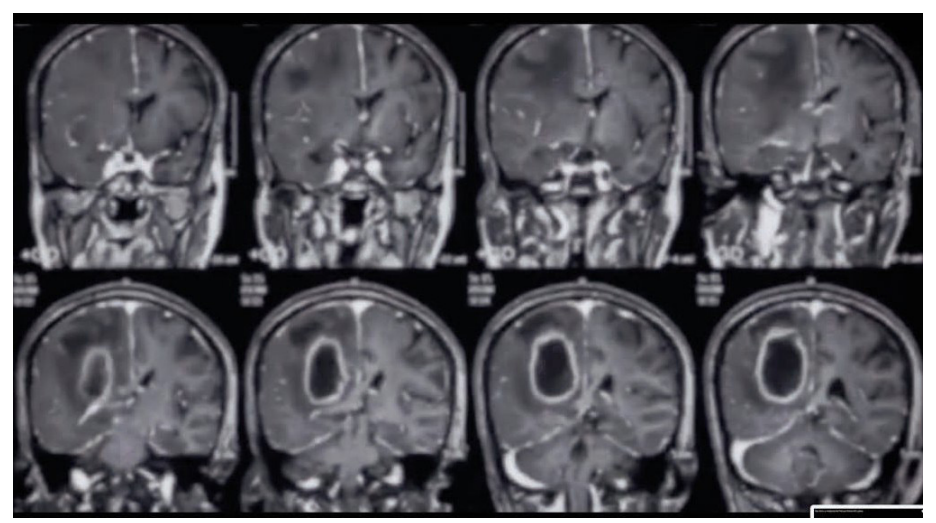

Trastornos neurológicos, que suelen presentarse en un grupo reducido de pacientes.

Rev. Med. Cine. 2020; 16(3), 235-238 Ediciones Universidad de Salamanca / @®@ J. Med. Mov., 2020; 16 (3), $235-238$ [238 ] 